\title{
A NOTE ON GAGLIARDO-NIRENBERG TYPE INEQUALITY
}

\section{XIAOFANG ZHOU}

School of Information Science and Technology

Jinan University

Guangzhou 510632

P. R. China

e-mail: zhouxf199108@163.com

\begin{abstract}
In this paper, we give a version of Gagliardo-Nirenberg type inequality associated to divergence elliptic operators. It is a generalized inequality based on the one established by [8].
\end{abstract}

\section{Introduction}

In [8], Kozono and Wadade proved the Gagliardo-Nirenberg type inequality with the critical index and related estimates in the Sobolev space. In fact, they proved the following Gagliardo-Nirenberg type inequality holds:

$$
\|u\|_{L^{q}\left(\mathbb{R}^{n}\right)} \leq C_{n} r^{\prime} q^{1 / r^{\prime}}\|u\|_{L^{p}}^{p / q}\left\|(-\Delta)^{\frac{n}{2 r}} u\right\|_{L^{r}\left(\mathbb{R}^{n}\right)}^{1-p / q},
$$

for all $u \in L^{p}\left(\mathbb{R}^{n}\right) \cap H^{n / r, r}\left(\mathbb{R}^{n}\right)$ with $1 \leq p \leq q<\infty, 1<r<\infty$, and $r^{\prime}=r /(r-1)$. Note that the constant $C_{n}$ in (1.1) depends only on $n$. Here $H^{s, r\left(\mathbb{R}^{n}\right)}$ denotes the homogeneous Sobolev space defined by 2010 Mathematics Subject Classification: 35K30, 42B35.

Keywords and phrases: Gagliardo-Nirenberg type inequality, divergence elliptic operators. Received July 8, 2015

(C) 2015 Scientific Advances Publishers 


$$
H^{s, r\left(\mathbb{R}^{n}\right)}=\left\{u \in S^{\prime}\left(\mathbb{R}^{n}\right):\left\|(-\Delta)^{s / 2} u\right\|_{L^{r}\left(\mathbb{R}^{n}\right)} \leq \infty\right\} .
$$

When $r \rightarrow \infty$, this leads to the following Gagliardo-Nirenberg type inequality:

$$
\|u\|_{L^{q}\left(\mathbb{R}^{n}\right)} \leq C_{n} r^{\prime} q^{1 / r^{\prime}}\|u\|_{L^{p}}^{p / q}\|u\|_{B M O}^{1-p / q}
$$

Naturally, we ask if in the inequality (1.1) the Laplace operators can be replaced by elliptic operators. In this paper, we consider the following elliptic operators:

$$
L=-\operatorname{div} A \nabla .
$$

In which $A=\left(a_{i, j}\right)_{n \times n}$ is a matrix of complex-valued, measurable functions satisfying the ellipticity conditions:

(1) $\lambda|\xi|^{2} \leq \operatorname{Re} \sum_{i, j} a_{i, j}(x) \xi_{i} \bar{\xi}_{j}=\operatorname{Re}<A \xi, \xi>$;

(2) $|<A \xi, \eta>| \leq \Lambda|\xi||\eta|$

where $0<\lambda \leq \Lambda<\infty$ and $\xi, \eta \in \mathbb{C}^{n}$. Such problem connects with so-called "square root problem", that is, if the following inequality holds:

$$
\left\|L^{1 / 2}(f)\right\|_{L^{2}\left(\mathbb{R}^{n}\right)} \leq c\|f\|_{L^{2}\left(\mathbb{R}^{n}\right)} .
$$

As we know, in [2], Auscher et al. solved it.

The problem also connect with the function spaces associated to such operators. For example, Duong and Yan in [5], [6] gave a characterization of Hardy space and BMO space associated to $L$ defined in (1.3).

In this paper, we replace the Laplace operators in (1.1) by $L$ and the traditional BMO spaces in (1.2) by $B M O_{L}$ spaces given in [6]. Thus we improve the results in [8]. Our methods mainly depend on the $H^{\infty}$ functional calculus theory in [9] and ideas in [8]. In Section 2, we give our main theorem and some preliminaries. In Section 3, we prove the main theorem. 
Throughout the paper, the constant "C" and "c" may be different somewhere, but it is not essential.

\section{Main Results and Important Lemmas}

In this paper, we give the following assumption:

Assumption. The holomorphic semigroup $e^{-z L},|\arg (z)|<\pi / 2-\theta$ is represented by the kernel $a_{z}(x, y)$, which satisfies the so-called Gaussian bound, that is, for all $\nu>\theta$

$$
\left|a_{z}(x, y)\right| \leq c_{\nu} h_{|z|}(x, y)
$$

for all $x, y \in \mathbb{R}^{n}$ and $|\arg (z)|<\pi / 2-\nu$, where $h_{t}(x, y)=\frac{1}{\mathbf{B}\left(x, t^{1 / 2}\right)}$ $e^{-|x-y|^{2} / t}$, in which $\mathbf{B}\left(x, t^{1 / 2}\right)$ denotes any ball with center $x \in \mathbb{R}^{n}$ and radius $t^{1 / 2}>0$.

Remark 2.1. In [1], Auscher and Tchamitchian found that if $\mathbf{A}$ is real, symmetric valued, the heat kernel $p_{t}(x, y)$ of the semigroup $e^{-t L}(t>0)$ satisfies the upper Gaussian bounds. If $\mathbf{A}$ is complex valued, the heat kernel $p_{t}(x, y)$ of the semigroup $e^{-t L}(t>0)$ may not satisfy the upper Gaussian bounds as $n \geq 5$. More details about the bound of $e^{-t L}$ for elliptic operators can be found in [1]. In fact, some other differential operators have the above similar properties, such as Schrödinger operators or degenerate elliptic operators, see [10].

In the following, we assume that $p_{t}(x, y)$ is the kernel of $P_{t}=e^{-t L}$, which can be seen as an approximation to identity. Set $Q_{t}=t L e^{-t L}$, then it can be proved that its kernel $v_{t}(x, y)$ also satisfies the Gaussian bound and Holder continuity estimates by using the Cauchy formula with the previous assumption. 
Moreover, we have $e^{-t L}(1)=1$ and $L e^{-t L}(1)=0$ under the assumption of $L$ (see Proposition 25 in [1]). Here for simplicity, we assume that $L$ is a self-adjoint operator.

By the previous assumptions about the accretivity condition on $L$, we can define the following square root operator and fractional integral operator by using spectral theory or $\mathbf{H}^{\infty}$ functional calculus theory in [9]

$$
\begin{gathered}
L^{1 / 2}=\frac{1}{\pi^{-1 / 2}} \int_{0}^{\infty} e^{-t L} L \frac{d t}{\sqrt{t}} \\
L^{-\alpha / 2}=\frac{1}{\gamma(\alpha)} \int_{0}^{\infty} e^{-t L} \frac{d t}{t^{1-\alpha / 2}}, \gamma(\alpha)=\pi^{n / 2} 2^{\alpha} \frac{\Gamma(\alpha / 2)}{\Gamma\left(\frac{n}{2}-\alpha / 2\right)} .
\end{gathered}
$$

It is not difficult to verify that

$$
\left|L^{-\alpha / 2} f(x)\right| \leq c I_{\alpha}(|f|)(x)
$$

where $I_{\alpha}$ is the classical fractional integral operator defined by

$$
I_{\alpha} f(x)=\int_{\mathbb{R}^{n}} \frac{f(y)}{|x-y|^{n-\alpha}} d y .
$$

Then we have (see also page 12 in [11]).

Proposition 2.1. Let L satisfy the previous assumptions. Then for $0<\alpha<n$,

(1) if $1<p<\frac{n}{\alpha}$ and $\frac{1}{q}=\frac{1}{p}-\frac{\alpha}{n}$, we have

$$
\left\|L^{-\alpha / 2} f\right\|_{L^{q}} \leq c\|f\|_{L^{p}}
$$

(2) if $\lambda>0$, we have

$$
\left|\left\{x \in \mathbb{R}^{n}:\left|L^{-\alpha} f(x)\right|>\lambda\right\}\right| \leq c\left(\frac{\|f\|_{L^{1}}}{\lambda}\right)^{q},
$$

where $\frac{1}{q}=1-\frac{\alpha}{n}$. 
We also need the following lemma:

Lemma 2.1. Let L satisfy the previous assumption. Then for all $t>0$

$$
\left\|e^{-t L} f\right\|_{L^{q}} \leq C t^{-\frac{n}{2}\left(\frac{1}{p}-\frac{1}{q}\right)}
$$

holds for $u \in L^{P}\left(\mathbb{R}^{n}\right), 1 \leq p \leq q \leq \infty$.

Proof. We can obtain the required $L^{p}-L^{q}$ estimates for heat semigroup by using the Gaussian bound of the heat kernel and the Young inequality.

Here we also need the following lemma:

Lemma 2.2. Let $L$ satisfy the previous assumption. Then for $0<\alpha<n$, $\left(I-e^{-t L}\right) L^{\frac{-\alpha}{2}}$ has an associated kernel $K_{\alpha, t}(x, y)$ which satisfies

$$
\left|K_{\alpha, t}(x, y)\right| \leq \frac{c \gamma(\alpha)^{-1}}{|x-y|^{n-\alpha}} \frac{t}{|x-y|^{2}}, \quad c>0 .
$$

Proof. This is just Lemma 5.3 in [4].

Define

$$
M\left(\mathbb{R}^{n}\right)=\left\{f \in L_{\mathrm{loc}}\left(\mathbb{R}^{n}\right): \int_{\mathbb{R}^{n}} \frac{|f(x)|}{1+|x|^{n+d}} d x<\infty, \text { for some } d>0\right\} .
$$

Next we define $B M O_{L}\left(\mathbb{R}^{n}\right)$.

Definition 2.1. We say $f \in M\left(\mathbb{R}^{n}\right)$ is of bounded mean oscillation associated with $L$ if

$$
\|f\|_{B M O_{L}}=\sup _{B(y, r)} \frac{1}{|B(y, r)|} \int_{B(y, r)}\left|f(x)-e^{-r^{2} L} f(x)\right| d x<\infty,
$$

where $B(y, r)$ is any ball in $\mathbb{R}^{n}$ with radius $r>0$ and center $y \in \mathbb{R}^{n}$.

Define the homogeneous Sobolev spaces associated to $L$ by

$$
\dot{H}_{L}^{s, r}\left(\mathbb{R}^{n}\right)=\left\{u \in S^{\prime}\left(\mathbb{R}^{n}\right):\left\|(-L)^{s / 2} u\right\|_{L^{r}\left(\mathbb{R}^{n}\right)}<\infty\right\} .
$$


In this paper, we will prove the following main results:

Theorem 2.1. Let L satisfy the previous assumptions. Then there exists a constant $C>0$ such that

$$
\|u\|_{L^{q}\left(\mathbb{R}^{n}\right)} \leq C r^{\prime} q^{1 / r^{\prime}}\|u\|_{L^{p}}^{p / q}\left\|L^{\frac{n}{2 r}} u\right\|_{L^{r}\left(\mathbb{R}^{n}\right)}^{1-p / q}
$$

for all $u \in L^{p}\left(\mathbb{R}^{n}\right) \cap \dot{H}_{L}^{n / r, r}\left(\mathbb{R}^{n}\right)$ with $1 \leq p \leq q<\infty, 1<r<\infty$, and $r^{\prime}=r /(r-1)$.

Let $r \rightarrow \infty$, we have the following version of the Gagliardo-Nirenberg type inequality for critical index:

Theorem 2.2. Let L satisfy the previous assumptions. Then there exists a constant $C>0$ such that

$$
\|u\|_{L^{q}\left(\mathbb{R}^{n}\right)} \leq C\|u\|_{L^{p}}^{p / q}\|u\|_{B M O_{L}\left(\mathbb{R}^{n}\right)}^{1-p / q}
$$

for all $u \in L^{p}\left(\mathbb{R}^{n}\right) \cap B M O_{L}\left(\mathbb{R}^{n}\right)$ with $1 \leq p \leq q<\infty$.

Now, we can give the following generalized John-Nirenberg inequality:

Corollary 2.1. Let $L$ satisfy the previous assumption. Then there exists a constant $C>0$ and $c>0$ such that

$$
\left|\left\{x \in \mathbb{R}^{n}:|u(x)|>t\right\}\right| \leq C \frac{\|u\|_{L^{1}}}{\|u\|_{B M O_{L}}} \frac{1}{\exp \left(\frac{c t}{\|u\|_{B M O_{L}}}\right)-1}
$$

hold for all $u \in L^{1} \cap B M O_{L}$ and $t>0$. In particular, we have

$$
\left|\left\{x \in \mathbb{R}^{n}:|u(x)|>t\right\}\right| \leq C \frac{\|u\|_{L^{1}}}{\|u\|_{B M O_{L}} \exp \left(-\frac{c t}{\|u\|_{B M O_{L}}}\right)},
$$

for all $u \in L^{1} \cap B M O_{L}$ and $t>\|u\|_{B M O_{L}}$. 


\section{The Proof of Main Results}

Proof of Theorem 2.1. For $u \in L^{p}\left(\mathbb{R}^{n}\right) \cap \dot{H}_{L}^{n / r, r}\left(\mathbb{R}^{n}\right)$, we have

$$
u(x)=e^{-t L} u(x)+\left(I-e^{-t L}\right) u(x)=e^{-t L} u(x)+\int_{0}^{t} \frac{d}{d r} e^{-r L} u(x) d r=u_{1}+u_{2}
$$

Set $m=\max (n+1, p, r)$ and $m \leq q<\infty$. Then according to Lemma 2.1, it yields

$$
\left\|u_{1}\right\|_{L^{q}} \leq c t^{-\frac{n}{2}\left(\frac{1}{p}-\frac{1}{q}\right)}\|u\|_{L^{p}}
$$

For the second term in (3.1), we have

$$
u_{2}(x)=\left(I-e^{-t L}\right) L^{-\frac{n}{2 r}} L^{\frac{n}{2 r}} u(x)=\int K_{\frac{n}{2 r}, t}(x, y) L^{\frac{n}{2 r}} u(y) d y
$$

By the Young inequality and Lemma 2.2, it follows

$$
\left\|u_{2}\right\|_{L^{q}} \leq c \gamma(n / r)^{-1}\left\|\frac{1}{|x-\cdot| \frac{n}{r^{\prime}}} \frac{t}{|x-\cdot|^{2}}\right\|_{L^{s}}\left\|L^{\frac{n}{2 r}} u\right\|_{L^{r}},
$$

where $\frac{1}{r^{\prime}}+\frac{1}{r}=1$ and $\frac{1}{r}+\frac{1}{s}=1+\frac{1}{q}$. Since $q \geq r$ and $q>n$, we have $s \geq 1$ and $n r^{\prime} /\left(n+r^{\prime}\right)<s<r^{\prime}$, and by using direct calculas

$$
\left\|\frac{1}{|x-\cdot| \frac{n}{r^{\prime}}} \frac{t}{|x-|^{2}}\right\|_{L^{s}} \leq c T^{1 / s} t^{-n /(2 q)}
$$

where $T=\frac{r^{\prime}}{r^{\prime}-s}+\frac{r^{\prime}}{\left(n+r^{\prime}\right) s-n r^{\prime}}$. Then, we have

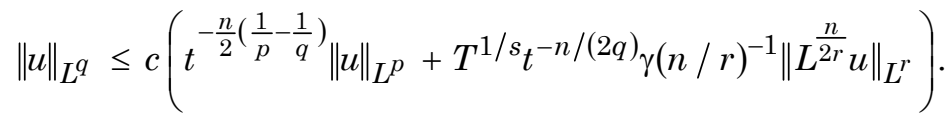


Choosing $t^{1 / 2}=\left(\frac{\left\|L^{n / 2 r} u\right\|_{L^{r}}}{\|u\|_{L^{p}}}\right)^{p / n}$, it gets

$$
\|u\|_{L^{q}} \leq c\left(1+T^{1 / s} \gamma(n / r)^{-1}\right)\|u\|_{L^{p}}^{p / q}\left\|L^{\frac{n}{2 r}} u\right\|_{L^{r}\left(\mathbb{R}^{n}\right)}^{1-p / q} .
$$

Next, the estimates of $T$ and the case of $p \leq q \leq m$ for the inequality is similar to the corresponding part in the proof of Theorem 2.1 in [8] with little modifications. Thus we omit its proof. This completes the proof of Theorem 2.1.

Use the notation $c B(x, r)=B(x, c r)$ for $c>0$. To prove Theorem 2.2, we need the following lemma:

Lemma 3.1. Let $\Omega$ be a relatively open subject of a fixed ball $B_{0}$ and suppose that $|\Omega| \leq\left|B_{0}\right| / 2$. Then there is a family of balls $\left\{B_{j}\right\}_{j \in Z^{+}}$so that

(1) The balls Bj are pairwise disjoint;

(2) $\Omega \subset \bigcup_{j} B_{j}^{*} \subset B_{0}$;

(3) $\left|\Omega \cap B_{j}^{*}\right| \leq \frac{1}{2}\left|B_{j}^{*}\right| \leq\left|\Omega^{c} \cap B_{j}^{*}\right|, j=1,2 \ldots$;

(4) $|\Omega| \leq \sum_{j}\left|B_{j}^{*}\right| \leq 2^{n+1}|\Omega|$,

where $B_{j}^{*}=2 B_{j}$.

Proof. This is just a variant version for balls of Lemma 7.2 in [3]. We omit its proof. Denote $u^{*}(t)$ as the rearrangement function of measurable function $u$, that is,

$$
u^{*}(t)=\inf \left\{\lambda: \sigma_{u}(\lambda) \leq t\right\}, \quad t>0,
$$


where the distribution function $\sigma_{u}(\lambda)$ is $|\{x:|u(x)|>\lambda\}|$. Denote by $u^{* *}$ the average function of $u^{*}$ defined by

$$
u^{* *}(t)=\frac{1}{t} \int_{0}^{1} u^{*}(t) d \tau, \quad t>0
$$

Suppose that $f \in M\left(\mathbb{R}^{n}\right)$ and $\Omega$ is an open set in $\mathbb{R}^{n}$, then define the sharp function associated to $L$ by

$$
f_{L, \Omega}^{\sharp}(x)=\sup _{x \ni B \subset \Omega} \frac{1}{|B|} \int_{B}\left|f(x)-e^{-r_{B}^{2} L} f(x)\right| d x, \quad x \in \Omega,
$$

otherwise, if $x \notin \Omega, f_{L, \Omega}^{\sharp}(x)=0$. Inspired by ideas of proof of Lemma 7.3 in [3], we prove the following lemma:

Lemma 3.2. Let $f$ be an integrable function supported on a ball $B_{0}$. Then

$$
f^{* *}(t)-f^{*}(t) \leq c\left(f_{L, B_{0}}^{\sharp}\right)^{*}(t), \quad\left(0 \leq t \leq \frac{\left|B_{0}\right|}{6}\right) .
$$

Proof. Without lose of generality, let $f$ be a nonnegative function. Fixed $0<t<\frac{\left|B_{0}\right|}{6}$, set

$$
E=\left\{x \in B_{0}: f(x)>f^{*}(t)\right\}, \quad F=\left\{x \in B_{0}:\left(f_{L, B_{0}}^{\sharp}\right)(x)>\left(f_{L, B_{0}}^{\sharp}\right)^{*}(t)\right\} .
$$

It is easy to know $|E| \leq t$ and $|F| \leq t$. Then there is a relatively open subset $\Omega \subset B_{0}$ with $|\Omega| \leq 3 t$ and $E \cup F \subset \Omega \subset B_{0}$. By Lemma 3.1, we obtain some open balls $\Gamma=\left\{B_{j}\right\}_{j}$ satisfying properties in Lemma 3.1.

Use the notation $f_{B}=\frac{1}{|B|} \int_{B} f(x) d x$. Then

$$
\begin{gathered}
t\left(f^{* *}(t)-f^{*}(t)\right) \leq \int_{E}\left(f(x)-f^{*}(t)\right) d x=\sum_{j=1}^{\infty} \int_{E \cap B_{j}}\left(f(x)-f^{*}(t)\right) d x \\
=\sum_{j}\left\{\int_{E \cap B_{j}}\left(f(x)-\left(e^{-r_{B_{j}}^{2} L} f\right)_{B_{j}}\right) d x\right\}+\sum_{j}\left|E \cap B_{j}\right|\left\{\left(e^{-r_{B_{j}}^{2} L} f\right)_{B_{j}}-f^{*}(t)\right\}=A+B .
\end{gathered}
$$


Notice that

$$
A \leq \sum_{j}\left\{\left|f(x)-e^{-r_{B_{j}}^{2} L} f(x)\right| d x\right\} .
$$

Let $\left(\sum_{j}\right)^{\prime}$ denote the sum over those $j$ for which $\left(e^{-r_{B_{j}}^{2} L} f\right)_{B_{j}}>f^{*}(t)$.

Notice that $\left|E \cap B_{j}\right| \leq\left|\Omega^{c} \cap B_{j}\right|$ and $f(u) \leq f^{*}(t)$ on $\Omega^{c}$, then

$$
B \leq\left(\sum_{j}\right)^{\prime} \int_{\Omega^{c} \cap B_{j}}\left(\left(e^{-r_{B_{j}}^{2} L} f\right)_{B_{j}}-f(u)\right) d u \leq \sum_{j}\left\{\int_{B_{j}}\left|f(x)-e^{-r_{B_{j}}^{2} L} f(x)\right| d x\right\} .
$$

Since $B_{j} \cap F^{c} \neq \emptyset$, set $x_{j} \in B_{j} \cap F^{c} \neq \emptyset$, then

$$
\begin{aligned}
t\left(f^{* *}(t)-f^{*}(t)\right) & \leq \sum_{j}\left|B_{j}\right|\left\{\frac{1}{B_{j}} \int_{B_{j}}\left|f(x)-e^{-r_{B_{j}}^{2} L} f(x)\right| d x\right\} \\
& \leq \sum_{j}\left|B_{j}\right|\left(f_{L, B_{0}}^{\sharp}\right)\left(x_{j}\right) \leq \sum_{j}\left|B_{j}\right|\left(f_{L}^{\sharp}, B_{0}\right)^{*}(t) .
\end{aligned}
$$

Due to $|\Omega| \leq 3 t$, we have

$$
t\left(f^{* *}(t)-f^{*}(t)\right) \leq c \Omega\left(f_{L, B_{0}}^{\sharp}\right)^{*}(t) \leq c t\left(f_{L, B_{0}}^{\sharp}\right)^{*}(t) .
$$

This completes the proof.

Define the weak $L^{\infty}\left(\mathbb{R}^{n}\right)$ space $W L^{\infty}\left(\mathbb{R}^{n}\right)$ by

$$
W L^{\infty}\left(\mathbb{R}^{n}\right)=\left\{u \in L_{l o c}\left(\mathbb{R}^{n}\right):\|u\|_{W L^{\infty}}=\sup _{t>0}\left(u^{* *}(t)-u^{*}(t)\right)<\infty\right\} .
$$

Now we can give the proof of Theorem 2.2.

Proof of Theorem 2.2. Firstly, we can obtain the following results form Lemma 3.2 by letting $t \rightarrow \infty$ :

$$
\|u\|_{W L^{\infty}} \leq c\|u\|_{B M O_{L}}
$$


Notice the following results (Lemma 3.2 in [8])

$$
\|u\|_{L^{q}}\left(\mathbb{R}^{n}\right) \leq C\|u\|_{L^{p}}^{p / q}\|u\|_{W L^{\infty}\left(\mathbb{R}^{n}\right)}^{1-p / q},
$$

for all $u \in L^{p}\left(\mathbb{R}^{n}\right) \cap W L^{\infty}\left(\mathbb{R}^{n}\right)$ with $1 \leq p \leq q<\infty$. By (3.3), we have

$$
\|u\|_{L^{q}}\left(\mathbb{R}^{n}\right) \leq C\|u\|_{L^{p}}^{p / q}\|u\|_{B M O_{L}\left(\mathbb{R}^{n}\right)}^{1-p / q}
$$

Hence this finishes the proof of theorem.

Proof of Corollary 2.1. The proof is similar to that of Corollary 2.2 in [8]. Here we omit proof.

\section{References}

[1] P. Auscher and P. Tchamitchian, Square root problem for divergence operators and related topics, Asterisque 249 (1998), 1-172.

[2] P. Auscher, S. Hofmann, M. Lacey, A. Mcintosh and Ph. Tchamitchian, The solution of the Kato square root problem for second order elliptic operators on $\mathbb{R}^{n}$, Ann. of Math. 156(2) (2002), 633-654.

[3] C. Bennett and R. Sharpley, Interpolation of Operators, 129, Pure and Applied Mathematics, Academic Press, Inc., Boston, MA, 1988.

[4] D. G. Deng, X. T. Duong, A. Sikora and L. X. Yan, Comparison of the classical BMO with the BMO spaces associated with operators and applications, Rev. Mat. Iberoam. 24(1) (2008), 267-296.

[5] X. T. Duong and L. X. Yan, Duality of Hardy and BMO spaces associated with operators with heat kernel bounds, Amer. J. Math. 18 (2005), 943-973.

[6] X. T. Duong and L. X. Yan, New function spaces of BMO type, John-Nirenberg inequality, interpolation and applications, Comm. Pure Appl. Math. 58 (2005), 1375-1420.

[7] M. Frazier and B. Jawerth, A discrete transform and decompositions of distributional spaces, J. Funct. Anal. 93 (1990), 34-170.

[8] H. Kozono and H. Wadade, Remarks on Gagliardo-Nirenberg type inequality with critical Sobolev space and BMO, Math. Z. 259(4) (2008), 935-950. 
[9] Alan Mcintosh, Operators which have an $H_{\infty}$-calculus, Miniconference on operator theory and partial differential equations, Proc. Center. Math. Analysis ANU, Canberra, 14 (1986), 210-231.

[10] Z. Shen, On fundamental solution of generalized Schrödinger operators, J. Funct. Anal. 167 (1999), 521-564.

[11] N. T. Varopoulos, L. Saloff-Coste and T. Coulhon, Analysis and Geometry on Groups, Cambridge Tracts in Mathematics, 100, Cambridge University Press, Cambridge, 1992. 